\title{
Inhibitory Effects of "Group A Saponin" and "Group B Saponin" Fractions from Soybean Seed Hypocotyls on Radical-Initiated Lipid Peroxidation in Mouse Liver Microsomes
}

\author{
Keiji Nishida, ${ }^{1, *}$ Yoshiji Ohta, ${ }^{1}$ Yasuhisa Araki, ${ }^{2}$ Madoka ITo, ${ }^{2}$ \\ Yoichi NAGAMURA, ${ }^{3}$ and Isao ISHIGURO ${ }^{1}$ \\ ${ }^{1}$ Department of Biochemistry, ${ }^{2}$ Department of Internal Medicine, \\ School of Medicine, and ${ }^{3}$ Department of Clinical Chemistry, \\ School of Health Sciences, Fujita Health University, \\ Toyoake 470-11, Japan
}

(Received June 4, 1993)

Summary We examined the inhibitory effects of "group A saponin" and "group B saponin" fractions, which were extracted and separated from soybean seed hypocotyls, on water-soluble 2,2'-azobis(2-amidinopropane) (AAPH)- and lipid-soluble 2,2'-azobis(2,4-dimethylvaleronitrile) (AMVN)-initiated lipid peroxidation reactions that were conducted with mouse liver microsomes. The simultaneous addition of the group A saponin fraction dose-dependently inhibited AAPH- or AMVN-initiated lipid peroxidation in microsomes more strongly than that of the group $B$ saponin fraction. The group A saponin fraction inhibited the AAPHinitiated lipid peroxidation with a lag phase, while it immediately blocked the AMVN-initiated lipid peroxidation. The group A saponin fraction inhibited microsomal AAPH-initiated lipid peroxidation even when added to the reaction mixture after the lag phase period. Microsomes pretreated with the group A saponin fraction showed inhibition of AAPH-initiated lipid peroxidation with a prolonged lag phase, and the saponin fraction-pretreated microsomes showed inhibition of the AMVN-initiated lipid peroxidation in which a lag phase was found. These results indicate that in mouse liver microsomes, the group A saponin fraction from soybean seed hypocotyls, which is present outside and/or near the microsomal membranes, inhibits AAPH-initiated lipid peroxidation by inhibiting the initiation and propagation of this reaction, while it prevents microsomal AMVN-initiated lipid peroxidation mainly by inhibiting the propagation of this reaction. In addition, the

\footnotetext{
*To whom correspondence should be addressed.
} 
present results indicate that the group A saponin fraction can inhibit AAPH- or AMVN-initiated lipid peroxidation in mouse liver microsomes by its presence within the membranes and/or by binding to them.

Key Words: soyasaponin, soybean seed hypocotyl, lipid peroxidation, antioxidant, liver microsome (mouse)

Soybean seed hypocotyls contain a large amount of saponins termed "group A saponin" and "group B saponin" $[1,2]$. It has been reported that these saponins have some physiological activities, including goitrogenic [3], antilipolytic [4], antioxidative [4-6], and hepatoprotective properties $[4,6]$. We showed earlier that the administration of fractions of crude saponins and saponin groups from soybean seed hypocotyls to mice following treatment of them with carbon tetrachloride $\left(\mathrm{CCl}_{4}\right)$ tends to prevent liver injury with depression of the increases in serum and liver lipid peroxide levels routinely observed in mice so treated [7] and that in isolated rat hepatocytes, the group A saponin fraction from soybean seed hypocotyls has an preventive effect on $\mathrm{CCl}_{4}$-induced liver injury [8]. We also reported that saponin group fractions obtained from soybean seed hypocotyls inhibit $\mathrm{CCl}_{4}$-dependent lipid peroxidation in mouse liver microsomes without a lag phase and without affecting the monooxygenase system that participates in $\mathrm{CCl}_{4}$ metabolism and that the inhibitory mode of saponin group fractions, especially the group A saponin fraction, on this lipid peroxidation is similar to that of $\alpha$-tocopherol, a well-known chain-breaking antioxidant [9]. Moreover, it was shown that $\mathrm{CCl}_{4}$-dependent lipid peroxidation is inhibited in mouse liver microsomes pretreated with the group A saponin fraction [9]. From these findings, it has been suggested that saponin group fractions from soybean seed hypocotyls contain a component(s) inhibiting the chain reaction of lipid peroxidation in a similar manner as $\alpha$-tocopherol and that the group A saponin fraction inhibits $\mathrm{CCl}_{4}$-derived free radical-initiated peroxidation in mouse liver microsomes by its presence within the membranes and/or by binding to them.

In this present study, we examined the inhibitory effects of group A saponin and group B saponin fractions from soybean seed hypocotyls on lipid peroxidation initiated by $2,2^{\prime}$-azobis(2-amidinopropane) (AAPH), a water-soluble radical initiator, and 2,2'-azobis(2,4-dimethylvaleronitrile) (AMVN), a lipid-soluble radical initiator, which are capable of generating peroxy radicals at constant and measurable rates $[10]$ in mouse liver microsomes.

\section{MATERIALS AND METHODS}

Animals. Six-week-old male ddy mice, weighing 25-30 g, were purchased from Shizuoka Laboratory Animal Center Co., Hamamatsu, Japan and were used in all experiments. 
Chemicals. AAPH, AMVN, ethylenediaminetetraacetic acid disodium salt (EDTA $2 \mathrm{Na}$ ), butylated hydroxytoluene (BHT), 2-thiobarbituric acid (TBA), and all other chemicals were purchased from Wako Pure Chemical Ind., Ltd., Osaka.

Soybean saponin groups. Extraction and fractionation of saponin groups from the seed hypocotyl of soybeans were carried out according to the method of Shiraiwa et al. $[11,12]$ with some modifications. Components in the hypocotyl $(20 \mathrm{~g})$ were extracted by refluxing with $70 \%$ ethanol. The extract was separated into the fraction of sugars and isoflavonoids and the fraction of crude saponins by ODS column chromatography (ODS-A 60-60/3.2 $\times 50 \mathrm{~cm}$, YMC Co., Ltd., Kyoto). Saponins were reextracted from the crude saponin fraction with $80 \%$ methanol. This reextracted saponin fraction was fractionated into group A saponin and group B saponin fractions by gel filtration on a column of Sephadex LH-20 (4.5 $\times$ $74 \mathrm{~cm}$, Pharmacia, Tokyo). Detection of saponins in the group A saponin and group B saponin fractions was carried out by thin-layer chromatography and high-performance liquid chromatography $[11,12]$. The yields of the group $A$ saponin and group B saponin fractions were 1.3 and $0.6 \%(w / w)$, respectively, of the starting material (hypocotyls). The group A saponin fraction consisted of soyasaponin $\mathrm{Ab}$ or acetyl-soyasaponin $\mathrm{A}_{1}(60 \%)$ and soyasaponin $A_{1}(40 \%)$, and the group B saponin fraction, of soyasaponin Ba or soyasaponin V (20\%), soyasaponin $\mathrm{Bb}$ or soyasaponin I (70\%), and other soyasaponins (soyasaponin $\mathrm{Bc}$ or II, $\mathrm{Bb}^{\prime}$ or III, and $\mathrm{Bc}^{\prime}$ or IV [10\%]). These saponins belonging to both groups are known to possess the fundamental structures as shown in Fig. 1 [11, 12]. In all saponins in group A saponin, the sugar chain is attached to the C-22 of the aglycone (soyasapogenol A).

Preparation of mouse liver microsomes. The liver of mice under ether anesthesia was removed after substantial perfusion with ice-cold $0.15 \mathrm{M} \mathrm{KCl}$

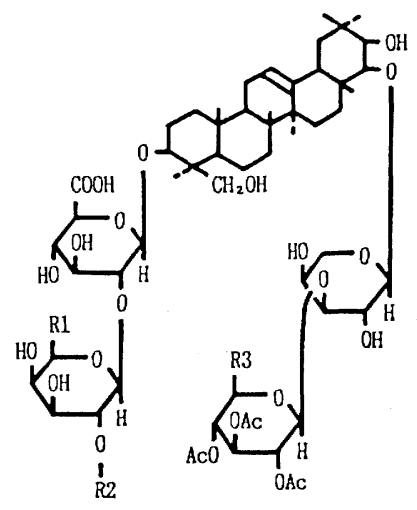

Group A saponin

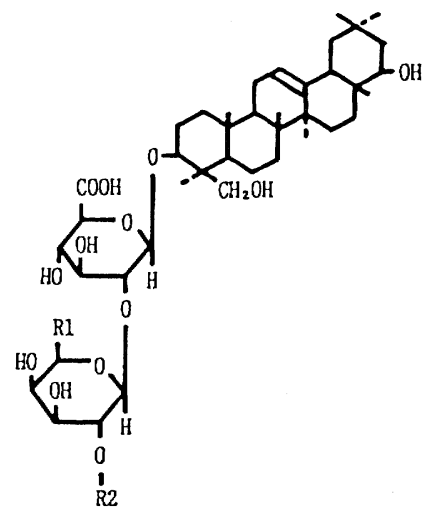

Group B saponin

Fig. 1. Fundamental structures of group A saponin and group B saponin. R1 represents $\mathrm{H}$ or $\mathrm{CH}_{2} \mathrm{OH}$; R2, H, $\beta$-D-glucose, or $\alpha$-L-rhamnose; and R3, $\mathrm{H}$ or $\mathrm{CH}_{2} \mathrm{OOCH}_{3}$ (see Refs. [11] and [12]). 
containing $1 \mathrm{~mm}$ EDTA-2Na. The liver was rinsed, weighed, and then homogenized in ice-cold $0.15 \mathrm{M} \mathrm{KCl}$ with a Potter-Elvehjem homogenizer. Liver microsomes were prepared by the method previously described [13]. The microsomal pellet was suspended in $0.15 \mathrm{M}$ Tris- $\mathrm{HCl}$ buffer ( $\mathrm{pH} 7.4$ ) containing $20 \%$ glycerol and stored at $-80^{\circ} \mathrm{C}$ until used. Microsomal protein was determined by the method of Lowry et al. [14], with bovine serum albumin used as a standard.

Induction of lipid peroxidation by radical initiators. AAPH ( $25 \mathrm{~mm})$ was added to the incubation medium containing $0.1 \mathrm{M}$ phosphate buffer $(\mathrm{pH} 7.5), 1 \mathrm{mM}$ EDTA $\cdot 2 \mathrm{Na}$, and microsomes $(0.1 \mathrm{mg}$ protein $/ \mathrm{ml})$. AMVN dissolved in methanol was evaporated to dryness under a stream of nitrogen. This AMVN was mixed with the suspension of liver microsomes and gently homogenized at $0^{\circ} \mathrm{C}$, to achieve a final concentration of $60 \mathrm{~mm}$ in the microsomes $(10 \mathrm{mg}$ protein $/ \mathrm{ml})$. The AMVN-treated microsomes, which had been kept on ice, were added to the same incubation medium as used for AAPH at a protein concentration of $0.1 \mathrm{mg} / \mathrm{ml}$ within 5 min after the AMVN treatment. The incorporation of AMVN into the membranes was demonstrated by centrifuging the microsomes and observing whether or not the supernatant solution was clear.

The reaction mixtures for AAPH- or AMVN-initiated lipid peroxidation were incubated in the presence or absence of $0.5,1.0$, or $2.0 \mathrm{mg} / \mathrm{ml}$ of either group A saponin or group B saponin fraction at $37^{\circ} \mathrm{C}$ in a shaking bath under aerobic conditions. The former fraction at a concentration of $0.5,1.0$, or $2.0 \mathrm{mg} / \mathrm{ml}$ was also added to the reaction mixture for AAPH-initiated lipid peroxidation 15 min after the onset of incubation.

Pretreatment of liver microsomes with group $\dot{A}$ saponin fraction. Liver microsomes $(10 \mathrm{mg}$ protein $/ \mathrm{ml})$ were incubated in the presence or absence of group A saponin fraction $(20 \mathrm{mg} / \mathrm{ml})$ at $37^{\circ} \mathrm{C}$ for $120 \mathrm{~min}$ in a shaking bath. The microsomes were washed with 5 vol. of ice-cold $0.15 \mathrm{M} \mathrm{KCl}$ and then centrifuged at $105,000 \times g$ for $60 \mathrm{~min}$ to remove the saponin fraction remaining in the medium. This washing was done two times. The microsomes pretreated with the saponin fraction were used for assay of AAPH- and AMVN-initiated lipid peroxidation reactions.

Lipid peroxidation assay. The activity of AAPH- or AMVN-initiated lipid peroxidation in microsomes was determined by the method of Buege and Aust [15] using the TBA reaction. The formation of the TBA-reactive products in the presence of BHT, an antioxidant, was monitored at $535 \mathrm{~nm}$. Malondialdehyde (MDA) formed was quantified by use of the molar extinction coefficient $\varepsilon=1.56 \times$ $10^{5} \mathrm{M}^{-1} \mathrm{~cm}^{-1}$ [15]. The various concentrations of group A saponin and group B saponin fractions used in this study did not affect the determination of MDA.

Statistical analysis. The results are expressed as mean \pm SE of three experiments. The statistical analysis was carried out by Student's $t$-test, and the level of significance was set at $p<0.05$. 


\section{RESULTS}

We first examined the effect of the simultaneous addition of group A saponin or group B saponin fraction from soybean seed hypocotyls on AAPH- or AMVNinitiated lipid peroxidation in mouse liver microsomes. The results are shown in Fig. 2. When liver microsomes were incubated with AAPH, a water-soluble radical initiator, for $45 \mathrm{~min}$, the amount of MDA formed was $9.83 \pm 0.44 \mathrm{nmol}$ $\mathrm{MDA} / \mathrm{mg}$ protein (Fig. 2A). The simultaneous addition of group A saponin fraction to the reaction mixtures caused a significant, dose-dependent inhibition of microsomal AAPH-initiated lipid peroxidation; the amounts of MDA produced in the presence of $0.5,1.0$, and $2.0 \mathrm{mg} / \mathrm{ml}$ of the group A saponin fraction were 70 , 42 , and $36 \%$, respectively, of the control value (Fig. 2A). In contrast, the simultaneous addition of the group $B$ saponin fraction at 0.5 and $1.0 \mathrm{mg} / \mathrm{ml} \mathrm{had} \mathrm{no}$ inhibitory effect on microsomal AAPH-initiated peroxidation, but the addition at $2.0 \mathrm{mg} / \mathrm{ml}$ did inhibit the peroxidation by $37 \%$ (Fig. 2A). When liver microsomes were incubated with $\mathrm{AMVN}$, a lipid-soluble radical initiator, for $120 \mathrm{~min}$, the amount of MDA formed was $6.18 \pm 0.21 \mathrm{nmol} \mathrm{MDA} / \mathrm{mg}$ protein (Fig. 2B). The simultaneous addition of group A saponin fraction at $0.5,1.0$, and $2.0 \mathrm{mg} / \mathrm{ml}$ inhibited microsomal AMVN-initiated lipid peroxidation by 20, 29, and 35\%, respectively (Fig. 2B). In contrast, the group B saponin fraction showed a $10 \%$ inhibition of microsomal AMVN-initiated lipid peroxidation, but only when 2.0 $\mathrm{mg} / \mathrm{ml}$ of the saponin fraction was added to the reaction mixture (Fig. 2B).

We further examined the time course of inhibition by the simultaneous addition of the group A saponin fraction on AAPH- or AMVN-initiated lipid
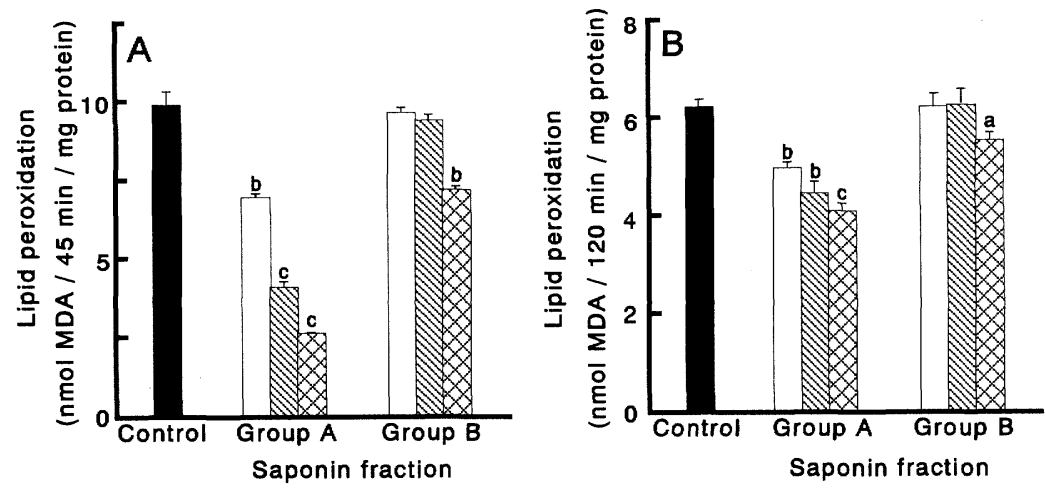

Fig. 2. Effect of the simultaneous addition of group A saponin or group B saponin fraction on AAPH (A)- or AMVN (B)-induced lipid peroxidation in mouse liver microsomes. The reaction mixtures containing microsomes $(0.1 \mathrm{mg}$ protein $/ \mathrm{ml})$ with $25 \mathrm{mM} \mathrm{AAPH}$ or $6 \mathrm{mM}$ AMVN were incubated for $45 \mathrm{~min}$ or $120 \mathrm{~min}$, respectively, in the presence of $0(\square), 0.5(\square), 1.0(\mathbb{\otimes})$, or $2.0(\mathbb{\otimes}) \mathrm{mg} / \mathrm{ml}$ of either group A saponin or group B saponin fraction as described in Materials and Methods. Each value represents the mean $\pm \mathrm{SE}$ of three determinations. Vs. control: $\mathrm{a}, p<0.05 ; \mathrm{b}, p<0.01 ; \mathrm{c}, p<0.001$. 
peroxidation in mouse liver microsomes. As shown in Fig. 3A, group A saponin fraction inhibited microsomal AAPH-initiated lipid peroxidation with a lag phase, and this phase was prolonged with increasing concentrations of the saponin
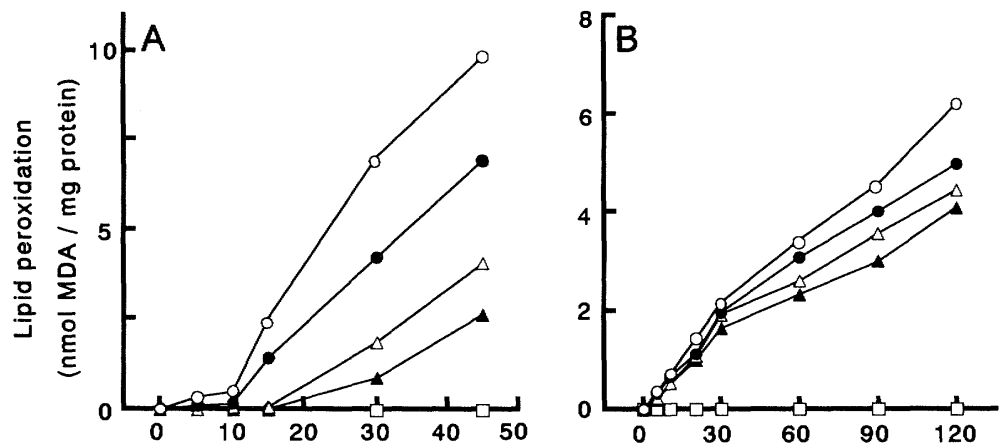

Incubation time $(\mathrm{min})$

Fig. 3. Effect of the simultaneous addition of group A saponin fraction on AAPH (A)- or AMVN (B)-initiated lipid peroxidation in mouse liver microsomes as a function of incubation time. The reaction mixtures containing microsomes $(0.1 \mathrm{mg}$ protein $/ \mathrm{ml})$ with either $25 \mathrm{~mm}$ AAPH or $6 \mathrm{~mm}$ AMVN were incubated in the presence of $0(0), 0.5$ $(\bullet), 1.0(\triangle)$, or $2.0(\Delta) \mathrm{mg} / \mathrm{ml}$ of group A saponin fraction; also the reaction mixtures containing microsomes $(0.1 \mathrm{mg}$ protein $/ \mathrm{ml})$ alone $(\square)$ were incubated as described in Materials and Methods. Each point represents the mean of three determinations.

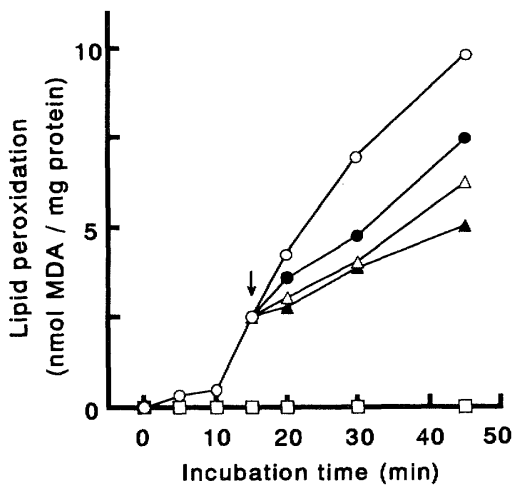

Fig. 4. Effect of the post-addition of group A saponin fraction on AAPH (A)- or AMVN (B)-initiated lipid peroxidation in mouse liver microsomes as a function of incubation time. The reaction mixtures containing microsomes $(0.1 \mathrm{mg}$ protein $/ \mathrm{ml})$ with $25 \mathrm{~mm}$ AAPH or $6 \mathrm{~mm}$ AMVN were incubated in the presence of $0(\bigcirc), 0.5(\bullet), 1.0(\triangle)$, or 2.0 $(\Delta) \mathrm{mg} / \mathrm{ml}$ of group A saponin fraction that was added $15 \mathrm{~min}$ after the onset of reaction; and the reaction mixtures containing microsomes $(0.1 \mathrm{mg}$ protein $/ \mathrm{ml})$ alone ( $\square$ ) were also incubated as described in Materials and Methods. Arrow indicates the time at which the saponin fraction was added. Each point represents the mean of three determinations. 


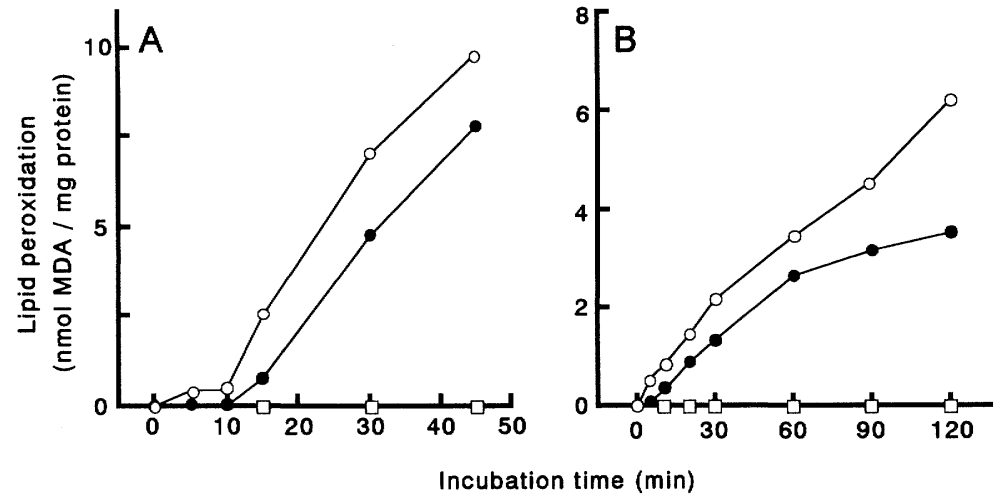

Fig. 5. Time course of AAPH (A)- or AMVN (B)-initiated lipid peroxidation in liver microsomes pretreated with and without group A saponin fraction. Liver microsomes $(0.1 \mathrm{mg}$ protein $/ \mathrm{ml})$ pretreated with $2.0 \mathrm{mg} / \mathrm{ml}$ of group A saponin fraction for $2 \mathrm{~h}(\bullet)$ or left untreated for this time $(0)$ were incubated with $25 \mathrm{~mm} \mathrm{AAPH}$ or $6 \mathrm{~mm}$ AMVN and the reaction mixtures containing microsomes $(0.1 \mathrm{mg}$ protein $/ \mathrm{ml})$ alone $(\square)$ were also incubated as described in Materials and Methods. Each point represents the mean of three determinations.

fraction added. In contrast, the fraction prevented microsomal AMVN-initiated lipid peroxidation without a lag phase (Fig. 3B).

When group A saponin fraction at $0.5,1.0$, or $2.0 \mathrm{mg} / \mathrm{ml}$ was added to the reaction mixture containing liver microsomes and AAPH after the conclusion of the lag phase, i.e., $15 \mathrm{~min}$ after the onset of the reaction, the saponin fraction inhibited AAPH-initiated lipid peroxidation without a time lag and in a dosedependent manner (Fig. 4).

Whether or not pretreatment of liver microsomes with group A saponin fraction would affect microsomal AAPH- or AMVN-initiated lipid peroxidation was examined by use of mouse liver microsomes pretreated with $2.0 \mathrm{mg} / \mathrm{ml}$ of group A saponin fraction for $2 \mathrm{~h}$. The results are shown in Fig. 5. Microsomes pretreated with group A saponin fraction elicited an apparent inhibition of AAPH-initiated lipid peroxidation with a prolonged lag phase (Fig. 5A), while the saponin fraction-pretreated microsomes exhibited inhibition of AMVNinitiated lipid peroxidation with a lag phase of about 5 min (Fig. 5B).

\section{DISCUSSION}

It has been demonstrated that water-soluble AAPH initiates lipid peroxidation in membranes by producing free radicals outside the membranes and that lipid-soluble AMVN induces lipid peroxidation in membranes by producing free radicals inside them [10]. The simultaneous addition of group A saponin fraction obtained from soybean seed hypocotyls was found to inhibit dose-dependently AAPH- or AMVN-initiated lipid peroxidation in mouse liver microsomes (Fig. 2).

Vol. 15, No. 3, 1993 
Although the simultaneous addition of $2.0 \mathrm{mg} / \mathrm{ml}$ of group B saponin fraction from soybean seed hypocotyls significantly inhibited both AAPH- and AMVNinitiated lipid peroxidation reactions, the inhibitory effect was weaker than that of the group A saponin fraction at $0.5 \mathrm{mg} / \mathrm{ml}$ (Fig. 2). From these findings, we conclude that the group A saponin fraction contains more potent saponin(s) than the group B saponin fraction for inhibiting lipid peroxidation initiated by free radicals generated in intra- or extramicrosomal membranes. The time course of inhibition by the simultaneous addition of the group A saponin fraction on AAPH- and AMVN-initiated lipid peroxidation reactions in mouse liver microsomes was examined to elucidate the inhibitory mode of the saponin fraction on lipid peroxidation initiated by these prooxidants. The group A saponin fraction inhibited the AAPH-initiated lipid peroxidation with a lag phase, while it reduced the AMVN-initiated lipid peroxidation without such a phase (Fig. 3). In addition, this fraction inhibited AAPH-initiated lipid peroxidation in mouse liver microsomes even when added to the reaction mixture after the conclusion of the lag phase (Fig. 4). These results indicate that group A saponin fraction from soybean seed hypocotyls can prevent microsomal lipid peroxidation initiated by free radicals generated outside the membranes by inhibiting the initiation and propagation of the reaction when the saponin fraction is present outside and/or near the membranes. These results also indicate that the group A saponin fraction can prevent microsomal lipid peroxidation initiated by free radicals generated inside the membranes mainly by inhibiting the propagation of the reaction when the saponin fraction exists outside and/or near the membranes.

Mouse liver microsomes pretreated with the group A saponin fraction had an inhibitory effect on AAPH- and AMVN-initiated lipid peroxidation reactions with a lag phase (Fig. 5). This result indicates that a saponin(s) present in the saponin fraction can exist within the membranes and/or bind to them and that the saponin fraction can prevent microsomal lipid peroxidation initiated by free radicals generated both inside and outside the membranes by inhibiting the initiation of the reaction when the saponin fraction is present in the membranes.

We demonstrated earlier that group A saponin from soybean seed hypocotyls inhibits $\mathrm{CCl}_{4}$-dependent lipid peroxidation in mouse liver microsomes in a similar manner as $\alpha$-tocopherol, a lipid-soluble chain-breaking antioxidant [9]. Palozza et al. [16] showed that in rat liver microsomes, $\alpha$-tocopherol inhibited AAPHinitiated lipid peroxidation with a lag phase and that although $\alpha$-tocopherol at 8 $\mathrm{nmol} / \mathrm{mg}$ protein inhibited AMVN-initiated lipid peroxidation in the liver microsomes with a lag phase, the vitamin at $4 \mathrm{nmol} / \mathrm{mg}$ protein blocked the peroxidation without a lag phase. Such similarities in the mode to inhibit liver microsomal lipid peroxidation between the group A saponin fraction and $\alpha$ tocopherol suggest that the former inhibits free radical-initiated lipid peroxidation in liver microsomes by breaking the chain reaction of the lipid peroxidation like $\alpha$-tocopherol.

Santiago et al. [17] have indicated that Japanese soybean paste or "miso" 
contains components that are able to scavenge directly hydroxyl and superoxide radicals. We have obtained unpublished data that group A saponin fraction from soybean seed hypocotyls reacts with 1,1-diphenyl-2-picrylhydrazyl, a free radical model that is known to react with $\alpha$-tocopherol [18]. From these findings, we speculate that group A saponin fraction can directly scavenge free radicals including oxygen radicals. Ohminami et al. $[4,6]$ reported that soyasaponin $\mathrm{A}_{1}$, belonging to group A saponin, inhibits lipid peroxidation in heated salad oil more strongly than other soyasaponins such as soyasaponin I (soyasaponin Bb), soyasaponin II (soyasaponin $\mathrm{Bc}$ ), and soyasaponin $\mathrm{A}_{2}$. Also, Tanizawa et al. [5] showed that soyasaponin $\mathrm{A}_{1}$ strongly inhibits the initiation of NADPH-dependent lipid peroxidation in mouse liver microsomes. The group A saponin fraction from soybean seed hypocotyls that was used in this study consists of soyasaponin $\mathrm{A}_{1}$ (40\%) and soyasaponin Ab (acetyl-soyasaponin A) (60\%). Further study is needed to determine which of these two is mainly responsible for the observed inhibition of AAPH- or AMVN-initiated lipid peroxidation in mouse liver microsomes.

In contrast, the inhibitory effect of the group B saponin fraction on lipid peroxidation initiated by AAPH or AMVN in mouse liver microsomes was weaker than that of the group A saponin fraction (Fig. 2). A similar difference between the two fractions was observed for liver microsomal $\mathrm{CCl}_{4}$-dependent lipid peroxidation [9]. Such a weak inhibitory effect of the group B saponin fraction on liver microsomal lipid peroxidation seems to be explained by the experiments of Rigy et al. [19], who reported no antioxidant activity of soyasaponin $\mathrm{Bb}$ (soyasaponin I), which accounts for $70 \%$ of the total saponins in the group B saponin fraction used in this study. These researchers assessed the antioxidant activity of soyasaponin $\mathrm{Bb}$ by using a Rancimat apparatus to determine its effect on the induction period of heat oxidation of pure refined lard that was free from natural and added antioxidants. In addition, this clear difference in inhibition of liver microsomal lipid peroxidation between group A saponin and group B saponin fractions from soybean seed hypocotyls seems to be explained based on the structural difference between the two groups because a sugar chain attached to the C-22 of the aglycone occurs in saponins of the group A saponin fraction, while it is not present in saponins of the group B saponin fraction [11,12] (see Fig. 1). Furthermore, this sugar chain in the group A saponin fraction may play a similar role as the phytyl side chain of $\alpha$-tocopherol, which is known to react directly with peroxy radicals [10], because the mode of action of the group A saponin fraction to inhibit liver microsomal lipid peroxidation is very similar to that of $\alpha$-tocopherol, as described above. However, these matters should be further studied.

In conclusion, the present results indicate that the group A saponin fraction obtained from soybean seed hypocotyls is able to act as an antioxidant in liver microsomes like $\alpha$-tocopherol.

We thank Dr. Y. Honda (Marusan Ai Co., Ltd., Okazaki, Japan) for his kind gift of group A saponin and group B saponin fractions from soybean seed hypocotyls.

Vol. 15, No. 3, 1993 


\section{REFERENCES}

1. Tani, T., Katsuki, T., Kubo, M., Arichi, S., and Kitagawa, I. (1985): Histochemistry. V. Soyasaponins in soybeans (Glycine max MERRILL, seed). Chem. Pharm. Bull., 33, 38293833.

2. Shiraiwa, M., Harada, K., and Ohkubo, K. (1991): Composition and content of saponins in soybean seed according to variety, cultivation year and maturity. Agric. Biol. Chem., 55, 324-331.

3. Kimura, S. (1984): The revelation of toxicity which is caused by some poisonous substances derived from foodstuffs and its modification under nutritional conditions. Yakugaku Zasshi, 104, 423-439.

4. Ohminami, H., Okuda, H., Yoshikawa, M., and Kitagawa, I. (1981): Effect of soyasaponins on lipid metabolism. Proc. Symp. WAKAN-YAKU, 14, 157-162.

5. Tanizawa, H., Sazuka, M., Takino, Y., Kitagawa, I., Yoshikawa, M., Hayashi, T., and Arichi, S. (1982): Inhibitory effect of soyasaponins on the increase of lipid peroxide induced by adriamycin (ADR) in mice. Proc. Symp. WAKAN-YAKU, 15, 119-122.

6. Ohminami, K., Okuda, H., Hami, T., Kitagawa, I., Yoshikawa, M., Arichi, S., and Hayashi, T. (1981): Effect of soyasaponins on liver injury. J. Jpn. Soc. Nutr. Food Sci., 34, 105-108.

7. Higuchi, T., Nishida, K., Nagamura, Y., Honda, Y., Ito, M., and Ishiguro, I. (1992): Preventive effects of saponins from hypocotyls of soybean on both in vivo and in vitro liver injury by carbon tetrachloride. Clin. Rep., 26, 257-263.

8. Nishida, K., Araki, Y., Nagamura, Y., Ohta, Y., Ito, M., and Ishiguro, I. (1993): Preventive effect of saponin fractions separated from hypocotyls of soybean on $\mathrm{CCl}_{4}$-induced injury in isolated rat hepatocytes. Med. Biol., 126, 87-90.

9. Nishida, K., Ohta, Y., Araki, Y., Higuchi, T., Ito, M., Nagamura, Y., and Ishiguro, I. (1993): Effects of "group A saponin" and "group B saponin" fractions from soybean seed hypocotyls on carbon tetrachloride-dependent lipid peroxidation in mouse liver microsomes. $J$. Med. Pharm. Soc. WAKAN-YAKU, 10, 54-60.

10. Niki, E. (1989): A study on the action of vitamin $\mathrm{E}$ as antioxidant. Vitamins, 63, 539-549.

11. Shiraiwa, M., Kudo, S., Shimoyamada, M., Harada, K., and Ohkubo, K. (1991): Composition and structure of "group A saponin" in soybean seed. Agric. Biol. Chem., 55, 315-322.

12. Shiraiwa, M., Harada, K., and Ohkubo, K. (1991): Composition and structure of "group B saponin" in soybean seed. Agric. Biol. Chem., 55, 911-917.

13. Ishiguro, I., Ohta, Y., Ogitsu, N., Ito, Y., Harata, J., and Shinohara, R. (1982): Effects of transferrin and ceruloplasmin on liver microsomal lipid peroxidation. Jpn. J. Clin. Chem., 11, 207-213.

14. Lowry, O.H., Rosebrough, N.J., Farr, A.L., and Randall, R.J. (1951): Protein measurement with the Folin phenol reagent. J. Biol. Chem., 193, 265-275.

15. Buege, J.A., and Aust, S.D. (1978): Microsomal lipid peroxidation. Methods Enzymol., 52, 302-310.

16. Palozza, P., Moualla, S., and Krinsky, N. (1992): Effects of $\beta$-carotene and $\alpha$-tocopherol on radical-initiated peroxidation of microsomes. Free Radical Biol. Med., 13, 127-136.

17. Santiago, A.L., Hiramatsu, M., and Mori, A. (1992): Japanese soybean paste miso scavenges free radicals and inhibits lipid peroxidation. J. Nutr. Sci. Vitaminol., 38, 297-304.

18. Blois, M.S. (1958): Antioxidant determinations by the use of a stable free radical. Nature, 181, 1199-1200.

19. Rigy, N.M., Price, K.R., Coxon, D.T., and Fenwick, G.R. (1987): Effects of soyasaponin I on the oxidation of corn oil and lard. J. Sci. Food Agric., 40, 157-164. 